\title{
Conductivity Measurement of E-textiles using a Microstrip Ring Resonator
}

\author{
Tien Manh Nguyen ${ }^{\# 1}$, Jae-Young Chung ${ }^{\# 2}$ \\ \# Dept. of Electrical \& Information Engineering, Seoul National Univ. of Sci. \& Tech., \\ 232 Gongneung-ro, Nowon-gu, Seoul, 139-743, Korea \\ ${ }^{1}$ manh.ngtien@gmail.com \\ 2 jychung@seoultech.ac.kr
}

\begin{abstract}
A conductivity measurement technique applicable for highly conductive textile materials is presented in this work. We employ a microstrip ring resonator as a test fixture to ease sample preparation and to increase measurement sensitivity. With this, the conductivity of a thin textile can be measured up to $10^{6} \mathrm{~S} / \mathrm{m}$ at $2 \mathrm{GHz}$. The conductivity value is extracted by comparing the measured and simulated transmission coefficients in an active learning iterative solver based on the surrogate-based optimization. The conductive textiles under test were Zelt and silver-coated e-textile, and the resulted conductivities are $2.29 \times 10^{5}$ and $3.47 \times 10^{5} \mathrm{~S} / \mathrm{m}$, respectively. These values are close to the DC conductivity given by the manufacturers.
\end{abstract}

Keyword- Conductivity, e-textile, microwave, ring resonator, surrogate-based optimization.

\section{INTRODUCTION}

Conductive textiles have been progressively used for designing wearable antennas in Wireless Body Area Network (WBAN) communications and Internet of Things (IoT) applications [1]-[2]. They are highly flexible, durable and restorable, allowing direct integration onto clothing by ordinary sewing or embroidering techniques. They also offer low sheet resistance suitable for efficient current transmission without significant ohmic loss.

The usual sheet resistance $\left(R_{s}\right)$ of conductive textiles in the market ranges from $0.04 \sim 0.4 \Omega /$ sq [3]. In other words, the conductivity (s) is $1.4 \times 10^{5}-1.3 \times 10^{6} \mathrm{~S} / \mathrm{m}$ based on the formula $\sigma=1 /\left(R_{s} t\right)$, where $t$ is the thickness of the textile. These values are measured at DC using the well-known four-point-probe or Van der pauw method. For a wearable antenna application, however, it is necessary to acquire $\sigma$ or $R_{s}$ in the microwave regime to promote accurate antenna modeling.

In contrast to copious measurement techniques for the permittivity of dielectrics, there is considerably less reported for the conductivity measurement of highly conductive materials $\left(\sigma>10^{5} \mathrm{~S} / \mathrm{m}\right)$ at microwave frequencies ( $>1 \mathrm{GHz}$ ) [3]-[4]. In these methods, resonators are mainly used as a test fixture to capture a slight variation in the quality factor (Q-factor) of low conductive loss materials. Furthermore, the resonators are often in a planar form to ease the measurement of thin foil or textile samples. The material's $\sigma$ is obtained by detecting the perturbation of Q-factor represented in a closed-form equation under the assumption that the radiation loss is trivial. However, in practice, the radiation loss of the half-opened planar resonator may mislead the extracted $\sigma$ and should not be overlooked.

As an alternative, full-wave simulation data can be used instead of the approximate closed-form equation to estimate $\sigma$. In a full-wave simulation tool, a resonator identical to the measurement is modeled, and then the simulation data is collected by varying the material property (e.g., $\sigma$ ) of a sample under test. Subsequently, the measured and simulation data are compared in an optimization algorithm to find the best fit. Recent reports have verified the effectiveness of this method for the measurement of the dielectric constant $\left(\varepsilon_{r}\right)$ and loss tangent $(\tan \delta)$ of an antenna substrate [5]-[6].

In this letter, we propose a conductivity measurement method employing a planar ring resonator. Due to its highly resonating nature at the $\mathrm{GHz}$ frequency band, the ring resonator has been previously used for the characterization of low-loss substrates [7]. As in Fig. 1, the planar ring is formed by the conductive textiles of interest. Besides, we use the surrogate-based optimization (SBO) [8] as an adaptive search algorithm to extract s of the ring from the measured and simulation responses. The material samples under test are aluminum foil, conductive Zelt fabric, and silver-coated e-textile. 


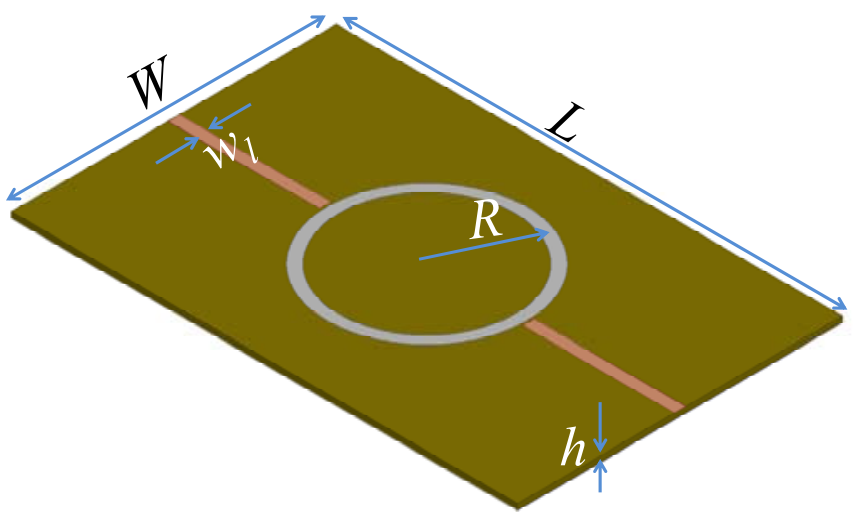

Fig. 1. Geometry of the microstrip ring resonator. The ring is made of conductive textile under interest.

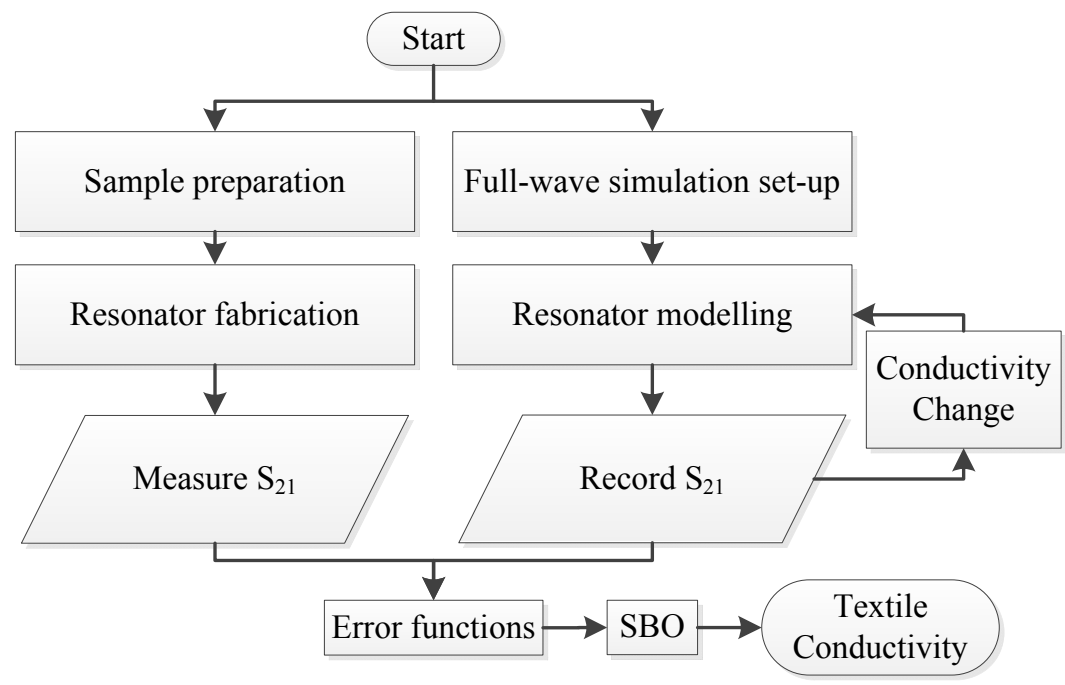

Fig. 2. Flow chart of the conductivity measurement process

\section{Conductivity Measurement Process}

Fig. 2 shows a flow chart of the conductivity measurement process. The microstrip ring resonator as in Fig. 1 is fabricated on a substrate and modeled in a full-wave simulation tool. More specifically, the resonator is fabricated on a FR-4 substrate with $h=1.6 \mathrm{~mm}, W=90 \mathrm{~mm}$, and $L=136 \mathrm{~mm}$. The transmission line in-between the ports and ring is made with copper. Otherwise, the ring is the material sample under interest. The radius of the ring is $R=25.9 \mathrm{~mm}$, and the width of the ring and transmission line is $w_{l}=3.2 \mathrm{~mm}$ to conform with $50 \Omega$ impedance matching condition. The gap between the ring and transmission line is $0.64 \mathrm{~mm}$.

In the simulation, the transmission coefficients $\left(S_{21}\right)$ are collected by varying $\sigma$ of the ring. Figure 3 shows the magnitude of $S_{21}$ by varying $\sigma$ from $10^{5}$ to $10^{8} \mathrm{~S} / \mathrm{m}$ interval of $10^{0.125}$. As can be seen, the peak is sharper and magnitude is higher (i.e., Q-factor is higher) as $s$ increases. In the meanwhile, the measured $S_{21}$ is compared with these sets of simulated $S_{21}$ using SBO to efficiently search for the $\sigma$ value that estimates the error function minimum. SBO is widely used to find solutions of non-linear electromagnetic problems as it offers an efficient iterative scheme by intelligently choosing the best sampling and evaluation strategies. We use the Matlab toolbox provided by Ghent University, Belgium [9]. This toolbox generates kriging models (i.e., Gauss regression model) based on approximate mapping between variables and error functions. The first kriging model is created by four sample points at the upper and lower bounds in the optimization range incorporating the Latin Hypercube Design (LHD) scheme. Subsequently, an adaptive sampling strategy called Expected Improvement (EI) is used to determine the next sample points with and to update the model.

In the SBO process, the error function should be carefully formulated to seek for an accurate result. The following four error functions are examined:

$$
\begin{aligned}
& E F_{1}=|Q-\bar{Q}| \\
& E F_{2}=\left|S_{21}^{r}\right|-\left|\overline{S_{21}^{r}}\right| \\
& E F_{3}=\left(\left|S_{21}^{r}\right|-\left|\overline{S_{21}^{r}}\right|\right)+\left(f^{r}-\overline{f^{r}}\right)
\end{aligned}
$$




$$
E F_{4}=\frac{1}{h-l+1}\left[\sum_{i=l}^{h}\left(\left|S_{21 i}^{r}\right|-\left|\overline{S_{21 i}^{r}}\right|\right)+\sum_{i=l}^{h}\left(\angle S_{21 i}^{r}-\angle \overline{S_{21 i}^{r}}\right)\right]
$$

, where and are loaded quality factors. and are the transmission coefficients of simulation and measurement at the resonant frequencies and, respectively. In case of $E F_{4}$, the arithmetic mean of $S_{21}$ magnitude and phase over the observation bandwidth (i.e., 41 frequency points in $40 \mathrm{MHz}$ ) are used as the variables. By this mean, $h$ and $l$ in EF4 are sample index numbers that are $20 \mathrm{MHz}$ higher and lower than the resonant frequency.

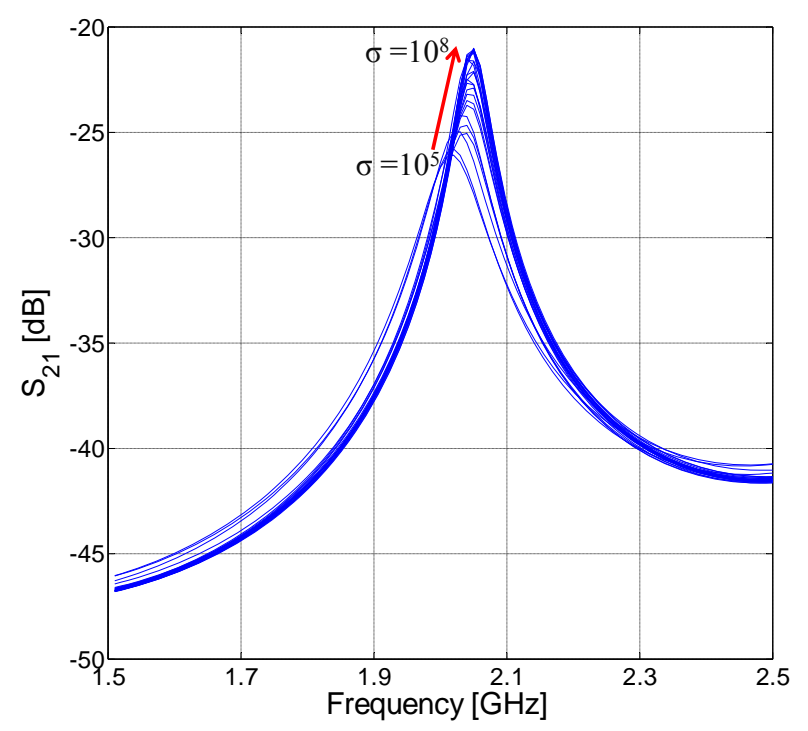

Fig. 3. $\mathrm{S}_{21}$ versus frequency as the conductivity of ring resonator changes

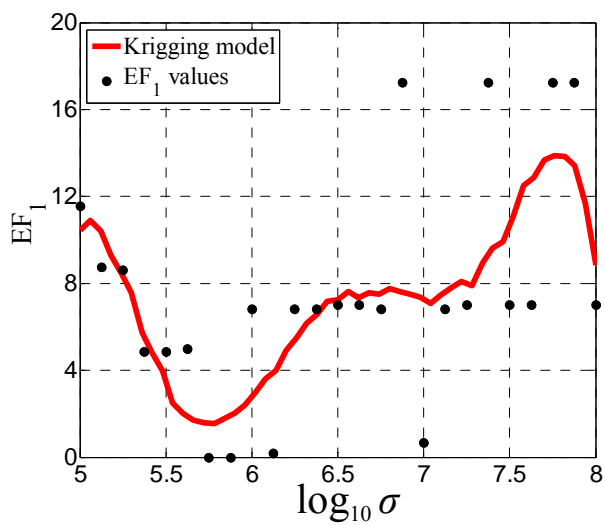

(a)

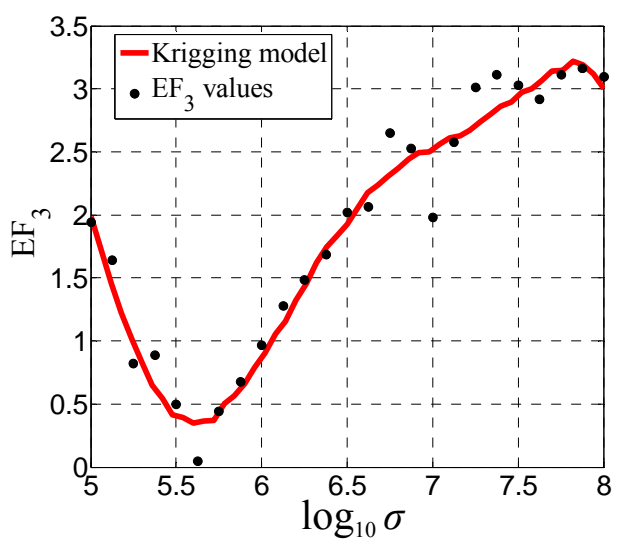

(c)

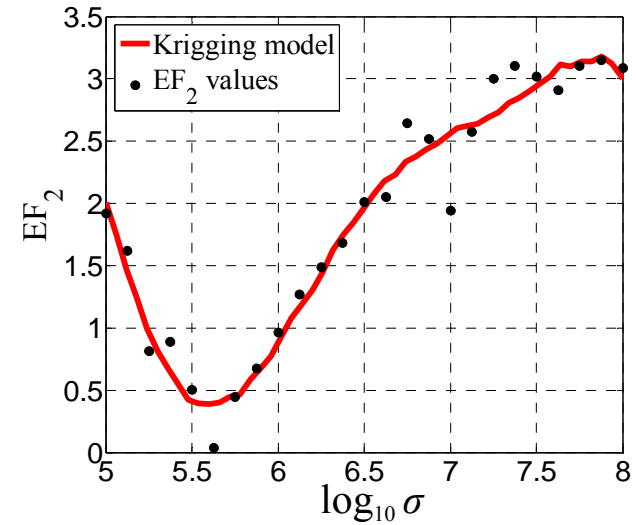

(b)

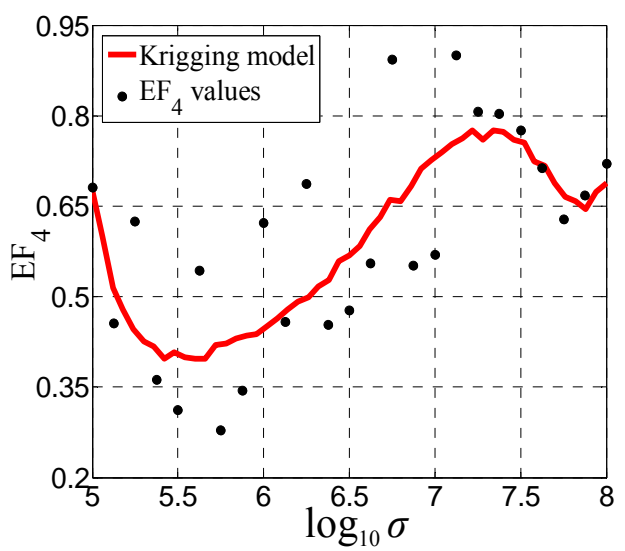

(d)

Fig. 4. Error function values and corresponding kriging models for (a) $E F_{1}$. (b) $E F_{2}$, (c) $E F_{3}$, and (d) $E F_{4}$. 
Fig. 4(a)-(d) shows the kriging models generated by using $E F_{1}, E F_{2}, E F_{3}$, and $E F_{4}$, respectively. In this simulation practice, $\sigma$ of the ring is assigned as $10^{5.6}=3.98 \times 10^{5} \mathrm{~S} / \mathrm{m}$, which is expected to be carried out at the end of the SBO process. The error function minima are located at $\sigma=10^{5.78}\left(6 \times 10^{5} \mathrm{~S} / \mathrm{m}\right), 10^{5.54}\left(3.47 \times 10^{5} \mathrm{~S} / \mathrm{m}\right)$, $10^{5.65}\left(4.47 \times 10^{5} \mathrm{~S} / \mathrm{m}\right)$, and $10^{5.6}\left(3.98 \times 10^{5} \mathrm{~S} / \mathrm{m}\right)$ for each error function. These results of indicate that $E F_{4}$ is the most effective one to evaluate $\sigma$, implying the change in $\sigma$ affects both the magnitude and phase of $S_{21}$. Thus, it is recommended to use the averaged $S_{21}$ magnitude and phase together in the error function to enhance the search accuracy in SBO. In the next section, $E F_{4}$ is used to obtain $\sigma$ of conductive textile materials along with the measured $S_{21}$ magnitude and phase.

\section{MEASUREMENT OF E-TEXTILES}

Four microstrip ring resonator are fabricated on FR-4 substrate with the same dimensions as in the previous section. Figure 5 depicts the fabricated resonators and measurement set-up. Four different rings are made from copper, aluminum foil, Zelt fabric, and silver-coated e-textile, and then placed in-between the two transmission lines.

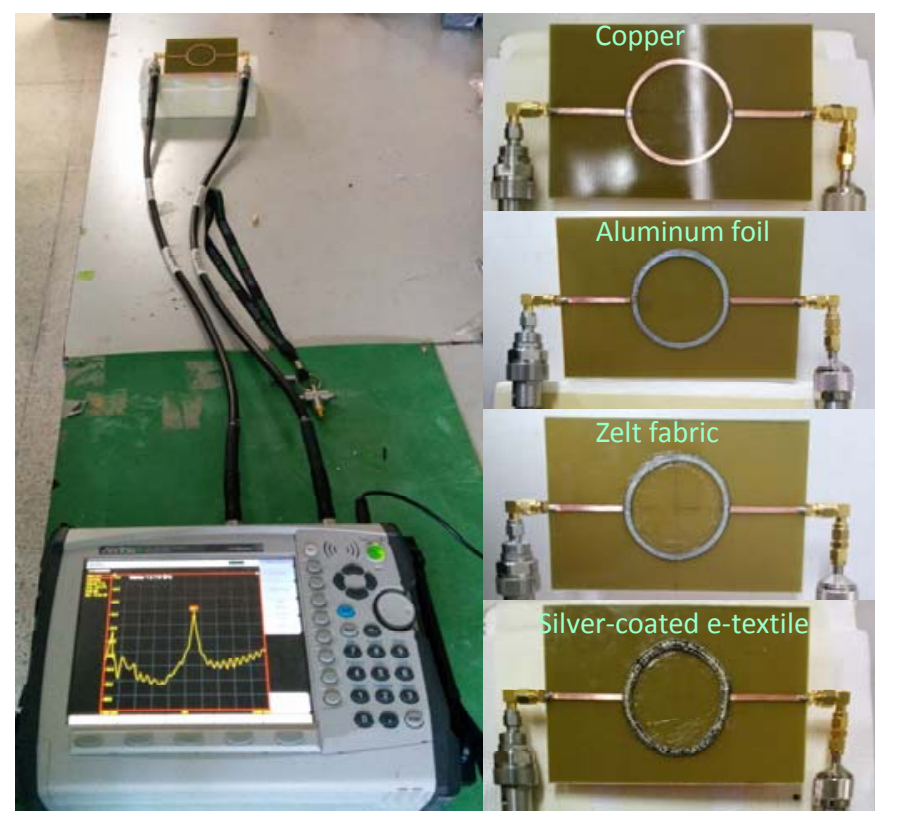

Fig. 5. Fabricated ring resonators and measurement set-ups with different ring conductors

Fig. 6 shows the measured $S_{21}$ magnitude with the four materials. The peak of the copper sample is the sharpest with highest magnitude followed by the aluminum foil. Having known the conductivity of copper as $\sigma$ $=5.8 \times 10^{7} \mathrm{~S} / \mathrm{m}$, we calibrate the other materials' $S_{21}$ against the copper's $S_{21}$ both in the measurement and simulation. With this, uncertainties due to connectors and test cables are calibrated out.

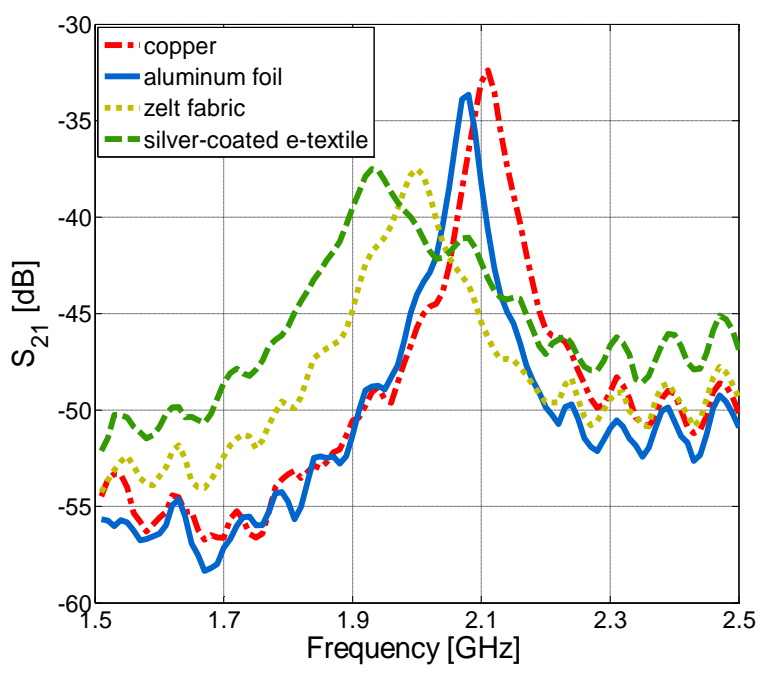

Fig. 6. Measured $S_{21}$ magnitude with the four materials. 
The calibrated $S_{21}$ data is processed in the SBO toolbox. The resulting kriging models based on $E F_{4}$ are shown in Fig. 7. For the aluminum foil, Zelt and silver-coated e-textile, the $E F_{4}$ minima is located at $\sigma=10^{5.84}$ $\left(6.92 \times 10^{5} \mathrm{~S} / \mathrm{m}\right), 10^{5.36}\left(2.29 \times 10^{5} \mathrm{~S} / \mathrm{m}\right)$, and $10^{5.54}\left(3.47 \times 10^{5} \mathrm{~S} / \mathrm{m}\right)$, respectively. It is noted that these measured conductivity values of the Zelt fabric and silver-coated e-textile are close to the conductivity provided by the manufacturers and previous works. The DC sheet resistance of the Zelt fabric is given by the manufacturer as $R_{S}<0.1 \Omega / \mathrm{sq}$ [10] indicating the DC conductivity is more than $10^{5} \mathrm{~S} / \mathrm{m}$ for a $0.1 \mathrm{~mm}$ thick sample. Another conductivity value of Zelt fabric shown in [11] is $1.749 \times 10^{5} \mathrm{~S} / \mathrm{m}$ which is close to the result obtained by the proposed method. The conductivity value of the silver-coated e-textile is reported as $1.8 \times 10^{5}$ $\mathrm{S} / \mathrm{m}[12]$, which presents relatively large difference to our measurement result, $\left(3.47 \times 10^{5} \mathrm{~S} / \mathrm{m}\right)$. This may be caused by the structural difference of the samples. For example, the measured sample in [12] is a sparsely sewed straight line, otherwise the ring here is woven with more density.

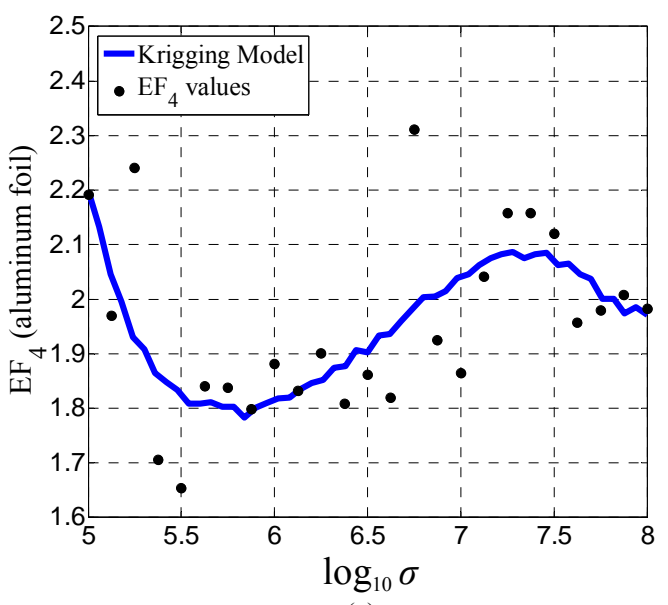

(a)

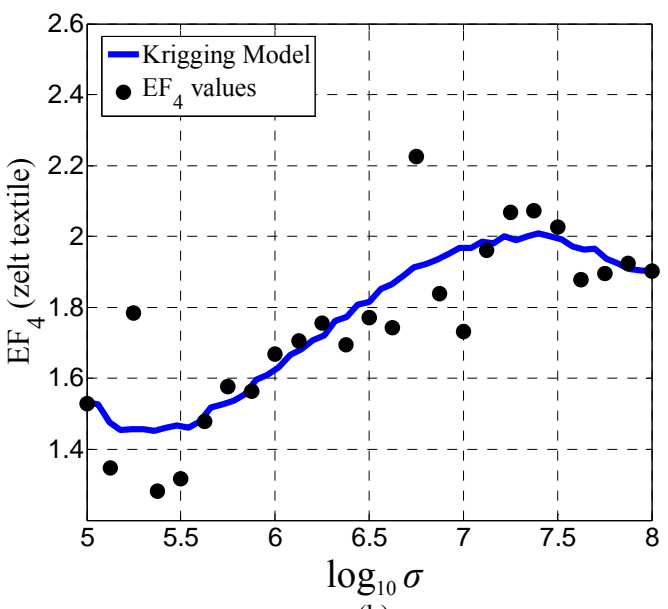

(b)

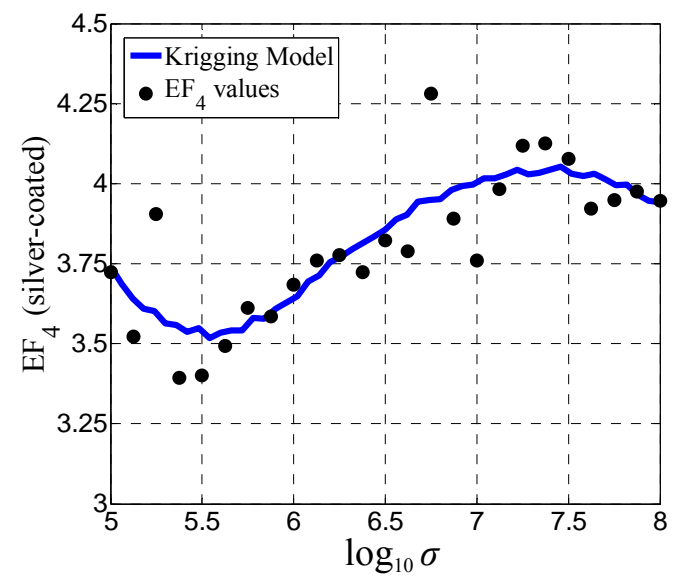

(c)

Fig. 7. $E F_{4}$ values with their SBO models for (a) aluminum ring, (b) zelt textile ring, and (c) silver-coated e-textile ring.

\section{IV.CONCLUSION}

A conductivity measurement procedure was described and demonstrated. The proposed method employs a microstrip ring resonator, full-wave simulation data in conjunction with SBO technique to measure the conductivity of thin textiles at the microwave frequency. In the SBO process, four error functions were evaluated, and it was found that the error function formulated by the averaged $S_{21}$ magnitude and phase was the most effective in extracting the conductivity value. The measured results of conductive Zelt fabric and silvercoated e-textile at $2 \mathrm{GHz}$ were close to the DC conductivity. Based on this, the conductive textiles composed of metal coated fibers can be regarded as non-dispersive, maintaining their high conductivity more than $10^{5} \mathrm{~S} / \mathrm{m}$ up to a few $\mathrm{GHz}$, suitable to implement wearable antennas.

\section{ACKNOWLEDGMENT}

This study was supported by the research program funded by the Seoul National University of Science and Technology. 


\section{REFERENCES}

[1] L. Zhang, Z. Wang, and J. L. Volakis, "Textile antennas and sensors for body-worn applications," IEEE Antennas Wireless Propag. Lett., vol. 11, pp. 1690-1693, 2012.

[2] A. Salman, E. Bicak, and Sezgin, "Wideband Antenna With Conductive Textile Radiators for a Dual-Sensor Subsurface Detection System,” IEEE Trans. Antennas Propag., vol. 59, no. 10, pp. 3746-3755, 2011.

[3] H. Shimasaki, T. Nakagawa, and M. Akiyama, "Measurement of the surface resistance of conductive textiles at microwave frequency," in IEEE Asia Pac. Microw. Conf., pp. 2580-2583, Dec., 2009.

[4] Y. Ouyang and W. Chappell, "Measurement of electrotextiles for high frequency applications," in Proc. IEEE MTT-S Int. Microw. Symp.,pp.1679-1682, 2005.

[5] F. Declercq, I. Couckuyt, H. Rogier, and T. Dhaene, "Environmental high frequency characterization of fabrics based on a novel surrogate modelling antenna technique," IEEE Trans. Antennas Propag., vol 61, no.10, pp. 5200-5213, 2013.

[6] P. M. Nguyen and J.-Y. Chung, "Material properties characterization based on measurements of reflection coefficient and bandwidth," J. Electromag. Eng. Sci., vol. 14, no. 4, pp. 382-386, 2014.

[7] A. Rashidian, M. T. Aligodarz, and D. M. Klymyshyn, "Dielectric characterization of materials using a modified microstrip ring resonator technique,” IEEE Trans. Dielectr. Electr. Insul., vol. 19, no. 4, 1392-1399, 2012.

[8] I. Couckuyt, F. Declercq, T. Dhaene, H. Rogier, and L. Knockaert, "Surrogate-based infill optimization applied to electromagnetic problems," Int. J. RF Microw. Comput.-Aided Eng., vol. 20, no. 5, pp. 492-501, Sep. 2010.

[9] Surrogate Modeling Lab, Available From: http://sumo.intec.ugent.be

[10] Material Connexion Company Website, Available From: http://ibrary.materialconnexion.com/

[11] M. I. Jais, M. F. Jamlos, M. F. Malek, and M. Jusoh, "Conductive E-textile analysis of $1.575 \mathrm{GHz}$ rectangular antenna with $\mathrm{H}$-slot for GPS application," in IEEE Loughborough Antennas Propag. Conf.,pp. 1-4, Nov., 2012.

[12] R. K. Shaw, B. R. Long, D. H. Werner, and A. Gavrin, "The characterization of conductive textile materials intended for radio frequency applications,” IEEE Antennas Propag. Mag., vol. 49, no. 3, pp. 28-40, 2007. 